\title{
Electrospun composite nanofibers containing nanoparticles for the programmable release of dual drugs
}

\author{
Yazhou Wang ${ }^{1}$, Weili Qiao ${ }^{1}$, Bochu Wang, Yiqiong Zhang, Pengyu Shao and Tieying Yin
}

In this study, electrospun composite nanofibers containing nanoparticles for the programmable release of dual drugs were successfully fabricated through a one-step, single-nozzle electrospinning technique. Field-emission scanning electron microscopy and scanning electron microscopy were used to examine the morphology of the nanoparticles and electrospun nanofibers, respectively. The distribution of nanoparticles in the composite nanofibers was evaluated by fluorescence microscopy and laser scanning confocal microscopy. The results indicate that the nanoparticles were encapsulated within the composite nanofibers, forming a core-sheath structure. The different release behaviors of the drugs were ascribed to their varying distribution in the nanofibers and their interaction with carriers. A programmable release of both drugs was also achieved by adjusting the preparation process of the electrospinning solution. Composite nanofibers that can provide a platform for dual-drug loading and the programmed release of each drug may find application in drug delivery systems or in the tissue-engineering field. Polymer Journal (2011) 43, 478-483; doi:10.1038/pj.2011.11; published online 2 March 2011

Keywords: composite nanofibers; core-sheath structure; dual drugs; electrospinning; programmable release

\section{INTRODUCTION}

In recent years, researchers have used electrospinning to fabricate ultrafine fibers with diameters in the nanometer range for wound dressing, tissue engineering scaffolds and drug delivery applications. ${ }^{1,2}$ Nanofibers exhibit a range of unique features and properties, such as the simplicity of their fabrication scheme, the diversity of materials suitable for processing into fibers, possession of high surface area and a complex pore structure, and they also have molecular-level alignment. ${ }^{3,4}$ Nanofibers with effective drug-loading capability and good stability have attracted much attention for their potential application in locally controlling drug release. They have been designed for the local and targeted delivery of anticancer drugs, antimicrobial agents, proteins and DNA. ${ }^{5}$ On the basis of different electrospinning techniques and nanofiber structures, drug loading is often achieved by three approaches: coating, embedding and encapsulating, which also affects the manner of drug release. ${ }^{6,7}$ So far most studies have focused on embedding a single drug species in nanofibers and controlling its release behavior, but an increasing number of studies have demonstrated that multi-drug loading and the programmable release of each drug are important in tissue engineering and disease therapy. ${ }^{8}$ For example, in cartilage repair, the sequential supplementation of growth factors was successfully performed to prevent the de-differentiation of cells: first, by promoting proliferation with one specific growth factor, followed by the differentiation and expression of a desired phenotype with another. ${ }^{9}$ In cancer therapy, cytotoxicity could be maximized if two anticancer drugs with distinct characteristics and action mechanisms were delivered simultaneously to the same cell. ${ }^{10}$ In addition, nanofibers have been proven effective in treating wounds by irrigation with anesthetic solution, followed by the application of prophylactic antibiotics to prevent wound infection. ${ }^{11}$ However, the successful incorporation of drugs with distinct properties, such as solubility, in nanofibers generally requires the use of multiple carriers or solvents, limiting the likelihood of simultaneous delivery. ${ }^{10}$ Furthermore, it is a great challenge to achieve controlled release of each drug in an intermittent and sequential manner. There have been very few studies on this aspect. ${ }^{8,9,12,13}$ In this paper, the electrospinning method was first used to construct nanoparticle-containing composite nanofibers that encapsulate dual drugs simultaneously and release each drug sequentially for topical drug delivery applications. In brief, we first prepared a set of drug-loaded nanoparticles, and then fabricated nanofibers to encapsulate the nanoparticles and another drug together through a one-step, single-nozzle electrospinning technique. The controlled and programmable release of two drugs could be achieved through their different distribution in the shell and core regions of the composite nanofibers, respectively.

Key Laboratory of Biorheological Science and Technology, Ministry of Education, Bioengineering College, Chongqing University, Chongqing, China

${ }^{1}$ These authors contributed equally to this work.

Correspondence: Dr B Wang, Key Laboratory of Biorheological Science and Technology, Ministry of Education, Bioengineering College, Chongqing University, Chongqing 400030, China.

E-mail: wangbc2000@126.com

Received 6 October 2010; revised 10 December 2010; accepted 20 December 2010; published online 2 March 2011 


\section{MATERIALS AND METHODS}

Materials

Poly ( $\varepsilon$-caprolactone) (PCL, $\mathrm{Mw}=80000)$ was purchased from Daigang Biotech (Jinan, China). Chitosan (CS, Mw $=100-300 \mathrm{kDa}$, degree of deacetylation $=85 \%$ ) and sodium tripolyphosphate (TPP, $\mathrm{Mw}=367.86)$ were obtained from Kelong Regent Factory (Chengdu, China). Rhodamine B was purchased from Sangon Biotech (Shanghai, China). Naproxen was purchased from Galaxy Chemical (Wuhan, China). Fluorescein isothiocyanate (FITC) was procured from Sigma-Aldrich (St Louis, MO, USA). Dimethyl sulfoxide and other solvents were analytical reagents.

\section{Preparation of drug-loaded CS nanoparticles}

CS nanoparticles were prepared on the basis of ionic gelation interaction between positively charged CS and negatively charged TPP. ${ }^{14}$ Briefly, they were prepared by adding $20-\mathrm{ml}$ TPP solution $\left(0.7 \mathrm{mg} \mathrm{ml}^{-1}\right)$ into $50-\mathrm{ml} \mathrm{CS}$ solution $\left(2.0 \mathrm{mg} \mathrm{ml}^{-1}\right)$ under slow mechanical stirring at room temperature. Rhodamine B-loaded CS nanoparticles were prepared using a CS solution containing the drug. Naproxen-loaded CS nanoparticles were prepared using a TPP solution containing the naproxen and $1 \%$ Tween-80, which was then added to the CS solution. Untrapped drug was removed, and drug-loaded nanoparticles were obtained by ultracentrifugation at 30000 r.p.m. for 50 min at $4{ }^{\circ} \mathrm{C} .{ }^{15}$

FITC-labeled nanoparticles were prepared according to a previously reported procedure ${ }^{16}$ Briefly, the precipitated nanoparticles were re-dispersed in 2-ml dimethyl sulfoxide solution, followed by the addition of $0.5 \mathrm{moll}^{-1}$ of $\mathrm{NaOH}$ to adjust the $\mathrm{pH}$ to $>6.5$. FITC was dissolved in dimethyl sulfoxide at a concentration of $2.5 \mathrm{mg} \mathrm{m}^{-1}$ and was slowly added to the suspension of CS nanoparticles. The reaction proceeded in the dark at room temperature under constant stirring for $3 \mathrm{~h}$. FITC-labeled nanoparticles were obtained by centrifugation.

\section{Preparation of electrospinning solutions}

Solution A was prepared by dissolving PCL in chloroform-methanol (3:1, v/v) to obtain a solution with a polymer concentration of $11 \mathrm{wt} \%$. A $1 \mathrm{wt} \%$ fraction of naproxen was added to a PCL solution under constant stirring for $2 \mathrm{~h}$ to obtain a uniform solution. Solution B was prepared according to the same method, but rhodamine B was used instead. Rhodamine B-loaded nanoparticles were re-dispersed in dimethyl sulfoxide and then added to solution A under constant stirring to obtain a uniform solution, which was marked solution C. Solution D was obtained by mixing naproxen-loaded nanoparticles and solution $\mathrm{B}$ uniformly. In solutions $\mathrm{C}$ and $\mathrm{D}$, the proportion of drug-loaded nanoparticles was $40 \%$. The components and amounts of solutions A, B, C and $\mathrm{D}$ are listed in Table 1.

\section{Electrospinning}

The experimental setup used for electrospinning consisted of a high-voltage power supply (DW-P503-4ACCD, Tianjin High Voltage Power Supply Company, Tianjin, China), a syringe pump (TJ-3A/W0109-1B, Longer Pump, Baoding, China), a syringe needle and an aluminum foil receptor. During electrospinning, a positive, $16 \mathrm{kV}$ charge was applied at the tip of the syringe needle. The electrospun fibers were collected on a piece of aluminum foil covered on an electrically grounded metal plate, which was placed at a distance of $12 \mathrm{~cm}$ behind the tip of the syringe needle. The mass flow rate was maintained at $1.5 \mathrm{mlh}^{-1}$. The electrospinning solutions prepared as described above, were directly used for electrospinning to obtain sets of nanofibers denoted as NF-A, NF-B, NF-C and NF-D, accordingly. ${ }^{17}$ The electrospinning setup and the preparation of nanoparticle-containing composite nanofibers are illustrated in Figure 1.

\section{Characterization of CS nanoparticles and nanofibers}

The micrographs of nanoparticle samples deposited on a copper grid were obtained using a field-emission scanning electron microscope (Nova 400, FEI, Hillsboro, Oregon, USA) operating at $10 \mathrm{kV}$. The size distribution of drugloaded nanoparticles was analyzed in a Zetasizer Nano ZS 90 (Malvern Instruments, Malvern, UK). A scanning electron microscope (TESCAN, VEGA II LMU, Brno, Czech) was used to observe the surface morphology of the electrospun composite nanofibers. Its accelerating voltage was $5.00 \mathrm{kV}$.
Table 1 Components and amounts of solutions A, B, C and D

\begin{tabular}{lcc}
\hline Solutions & Drug (1\%) & Drug-loaded nanoparticles \\
\hline A & Naproxen & - \\
B & Rhodamine B & - \\
C & Naproxen & Rhodamine B-loaded nanoparticles (40\%) \\
D & Rhodamine B & Naproxen-loaded nanoparticles (40\%) \\
\hline
\end{tabular}

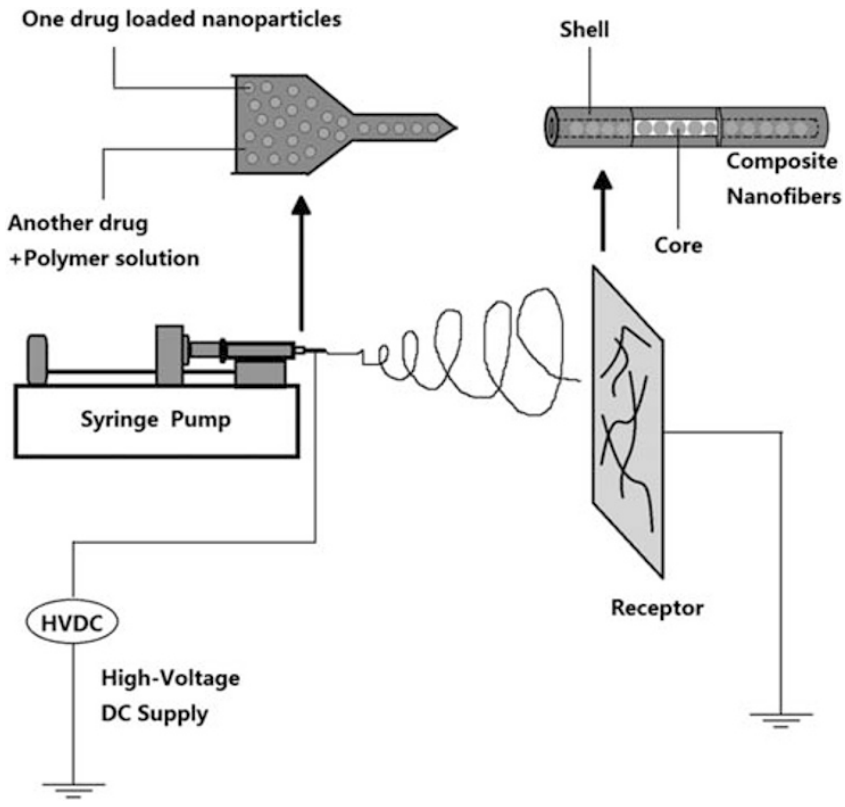

Figure 1 Schematic illustration of the electrospinning setup and the preparation of nanoparticle-containing composite nanofibers.

Fluorescence microscopy (DMT4000B, Leica, Wiesbaden, Germany), laser scanning confocal microscopy (LSCM, TCS SP5, Leica) and optical microscopy (XDS-1B, Chongqing Optec Instrument, Chongqing, China) were used to evaluate the distribution and quantification of rhodamine B and FITC in nanoparticles in the electrospun composite nanofibers.

\section{Release behaviors of model drugs from nanoparticles and nanofibers}

The nanofiber mats were cut into pieces of $100 \mathrm{mg}$ mass and placed in a vial filled with $20 \mathrm{ml}$ of pre-warmed phosphate buffer saline (PBS, pH 7.4). The nanoparticles, which were dispersed in 5-ml PBS and then enclosed in dialysis bags, were incubated in 20-ml PBS. The vial (protected from light) was incubated at $37^{\circ} \mathrm{C}$ in a thermostatted shaker (Boxun Industry \& Commerce Medical Equipment Factory, Shanghai, China). At appropriate intervals, $2 \mathrm{ml}$ of release medium was removed for sampling and replaced with an equal volume of fresh medium. The concentrations of the rhodamine B and naproxen released from the nanoparticles were determined by ultraviolet spectrometry. The concentrations of dual drugs released from the composite nanofibers were determined by dual-wavelength spectrophotometry at the same time; rhodamine B was quantified at $554 \mathrm{~nm}$, naproxen at $330 \mathrm{~nm}$ and the reference at $369 \mathrm{~nm}$ (The Appendix in the Supplementary Information shows the ultraviolet calibration curves of rhodamine B and naproxen in PBS).

\section{RESULTS AND DISCUSSION}

Size and morphology of CS nanoparticles

CS nanoparticles were prepared on the basis of the ionic gelation interaction between the negatively charged phosphate groups of TPP 
Table 2 Three states of different concentrations of CS and TPP reaction

\begin{tabular}{lccccc}
\hline & \multicolumn{5}{c}{ CS concentration $\left(\mathrm{mg} \mathrm{m}^{-1}\right)$} \\
\cline { 2 - 6 } TPP concentration $\left(\mathrm{mg} \mathrm{m}^{-1}\right)$ & 0.5 & 1.0 & 1.5 & 2.0 & 2.5 \\
\hline 0.25 & - & - & - & - & - \\
0.5 & $\downarrow$ & $\odot$ & $\odot$ & $\odot$ & $\odot$ \\
0.75 & $\downarrow$ & $\downarrow$ & $\odot$ & $\odot$ & $\downarrow$ \\
1.0 & $\downarrow$ & $\downarrow$ & $\downarrow$ & $\downarrow$ & $\downarrow$ \\
\hline
\end{tabular}

Abbrevitions: -, Clear solution; $\odot$, Opalescent suspension; $\downarrow$, Precipitation; CS, chitosan; TPP, tripolyphosphate.

and positively charged aminium group of CS, resulting in a spontaneous phase separation due to the electrostatic interaction when oppositely charged macromolecules are mixed together under magnetic stirring. ${ }^{14,18}$ The ionic gelation interaction was dependent on the ratio of the oppositely charged groups, and the nanoparticles were formed only at specific concentrations of CS and TPP. ${ }^{19}$ Thus, it was necessary to investigate the zone of particle formation first. CS was dissolved in $0.5 \%$ acetic acid solution to form solutions with various concentrations: $0.5,1.0,1.5,2.0$ and $2.5 \mathrm{mg} \mathrm{ml}^{-1}$. The $\mathrm{pH}$ value of these solutions was adjusted to 4.3. TPP was dissolved in water at various concentrations: $0.25,0.5,0.75$ and $1.0 \mathrm{mg} \mathrm{ml}^{-1}$. TPP solutions were added into CS solutions at a ratio of 2:5 (v/v) under magnetic stirring at room temperature. Three states appeared; the results are summarized in Table 2. A clear solution was observed when both the CS and TPP concentrations were excessively small, whereas aggregations were formed when they were excessively large. Opalescent suspensions represented a suspension of colloidal particles. Furthermore, an orthogonal design method was adopted to screen the optimal concentrations of CS and TPP using encapsulation efficiency and particle size as indicators. As a result, the concentrations of CS and TPP were 2.0 and $0.7 \mathrm{mg} \mathrm{ml}^{-1}$, respectively.

The mean size and size distribution of nanoparticle suspensions were analyzed using dynamic light scattering. The size distribution profile, shown in Figure 2a, represented the rhodamine B-loaded nanoparticles with a mean diameter of $408 \mathrm{~nm}$ and a narrow size distribution. There were two peaks in the size distribution profile of naproxen-loaded nanoparticles in Figure 2b, which was different from that observed for the rhodamine B-loaded particles. During the preparation of naproxen-loaded nanoparticles, Tween-80 was added to dissolve the insoluble naproxen. The large peak represented the size distribution of the naproxen-loaded nanoparticles, whereas the small one may indicate the size distribution of micelles composed of naproxen and Tween-80. The field-emission scanning electron microscope images exhibited roughly spherical nanoparticles (Figures $2 \mathrm{c}$ and d), and their size was in good agreement with the results obtained from the dynamic light scattering measurements.

\section{Nanoparticle-containing composite nanofibers}

Smooth nanofibers were fabricated by electrospinning from PCL solution without nanoparticles, as shown in Figure 3a. It was shown that composite nanofibers obtained from the composite solution containing nanoparticles were core-sheath structures with CS nanoparticles as cores and PCL as shells (Figures $3 \mathrm{~b}$ and c). The CS nanoparticles were wrapped by the polymer without being exposed on the surface of the fibers, even when they were larger than the nanofibers. ${ }^{8,20}$ The feasibility of incorporating nanoparticles (both inorganic and organic) into fibers by means of electrospinning for the
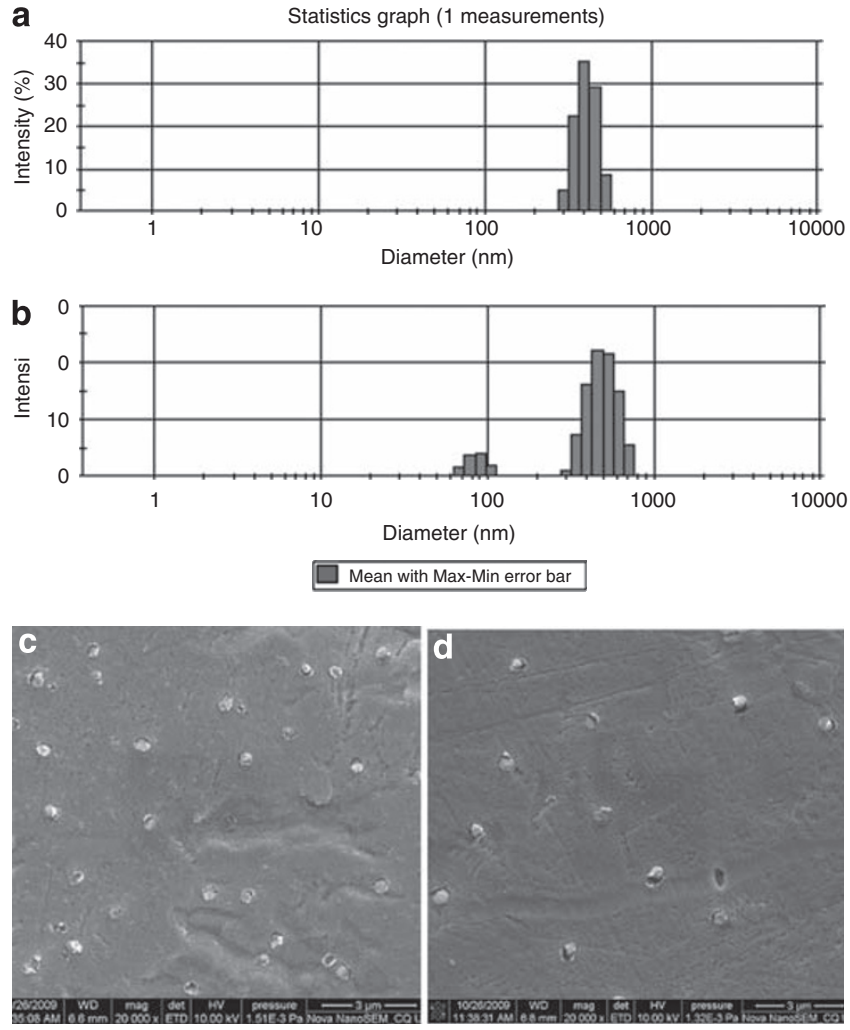

Figure 2 Size distribution of nanoparticles embedded with rhodamine $B$ (a) and naproxen (b). Their average sizes were about 408 and $495 \mathrm{~nm}$, respectively. (c) and (d) Show field-emission scanning electron microscope images of the two-drug-loaded chitosan nanoparticles, respectively.

preparation of nanocomposites has also been confirmed. ${ }^{21-23}$ When the suspension flowed through a long capillary and formed rapidly expanding and bending fluid jets, the dispersed phase had the tendency to accumulate in the center of the liquid to produce an elongation effect along the direction of the fluid during its flight in the air. This helped nanoparticles settle inside the fibers rather than on their surfaces. ${ }^{20}$ With the help of the surfactant Tween-80, which was able to decrease the interfacial energy and increase the interfacial area between the two phases, CS nanoparticles could be effectively re-dispersed in PCL solution, and ultimately, stable composite suspensions were obtained. ${ }^{1}$ The good compatibility and homogeneous dispersion of nanoparticles within the polymer solution could also assist in the encapsulation of the nanoparticles by the fibers, although there was still some aggregation of nanoparticles. ${ }^{8,24}$

Figure 4 showed an optical image of the nanoparticles encapsulated by the electrospun composite nanofibers. The encapsulated nanoparticles were visible under a bright-field optical microscope (Figure 4a). ${ }^{13}$ The white and bright spots indicated CS nanoparticles or their aggregates, distributed uniformly in the composite nanofibers. To gain further information about the distribution of the nanoparticles in the composite nanofibers, rhodamine B-loaded and FITClabeled CS nanoparticles were used and observed by fluorescence microscopy; they showed a favorable distribution, which can be seen in Figures $4 b$ and $c{ }^{25}$ Because of the specific reaction between the isothiocyanate group and the primary amine group of the D-glucosamine residue, FITC was easily attached to the CS nanoparticles. ${ }^{16}$ LSCM imaging revealed the core-shell structure of the composite nanofibers, which consisted of a PCL shell and an array of CS 

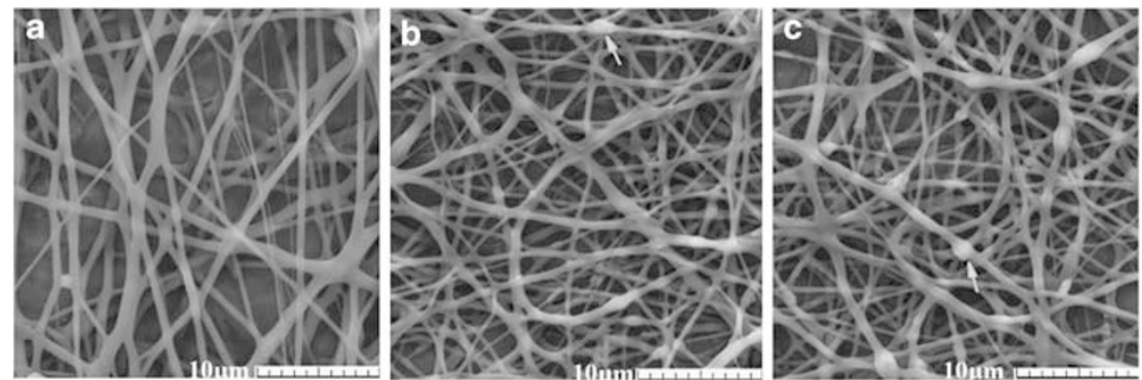

Figure 3 Scanning electron microscopy images of electrospun nanofibers. (a) Nanofibers without nanoparticles; (b) composite nanofibers with rhodamine Bloaded chitosan (CS) nanoparticles; (c) composite nanofibers with naproxen-loaded CS nanoparticles.
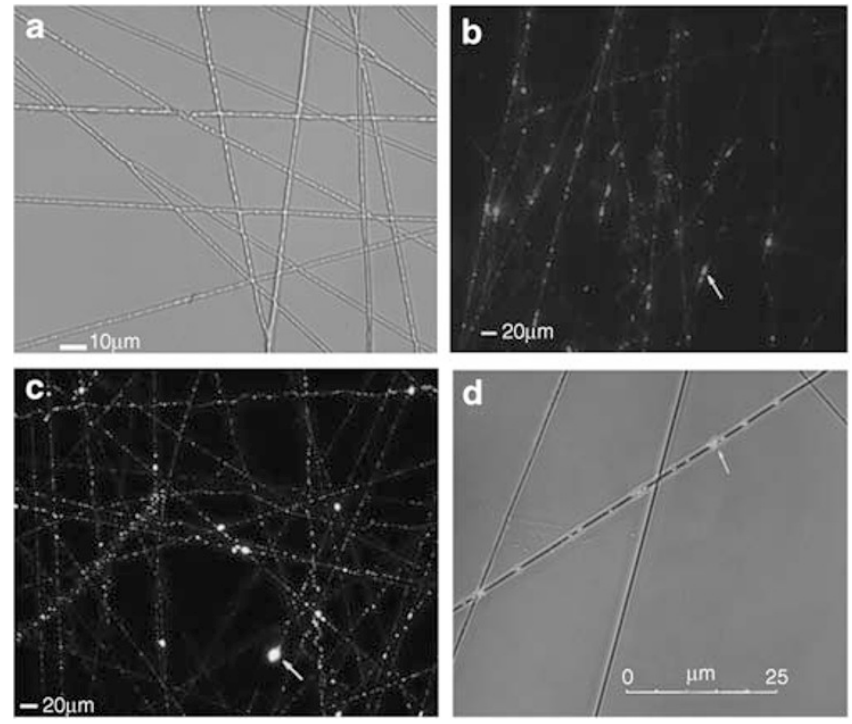

Figure 4 Electrospun composite nanofibers containing chitosan nanoparticles: (a) optical image; (b) and (c) fluorescence images of composite nanofibers containing rhodamine $B$ and Fluorescein isothiocyanate (FITC-labeled nanoparticles, respectively; (d) laser scanning confocal microscopy image.

nanoparticles as the core (Figure $4 \mathrm{~d}$ ). They were linearly packed and aligned along the axes of the fibers, which could be easily achieved by the application of shear force during the electrospinning process. ${ }^{8,17,26}$

\section{In vitro drug release}

The release behaviors of rhodamine $\mathrm{B}$ and naproxen from the composite nanofibers NF-C and NF-D were studied and were shown in Figure 5. It was observed that naproxen was released much faster than rhodamine B from composite NF-C. At $72 \mathrm{~h}$, the accumulated drug-release rate reached 60.53 and $18.15 \%$ for naproxen and rhodamine $\mathrm{B}$, respectively (Figure $5 \mathrm{a}$ ). We can infer that the release behaviors of the two drugs should be dependent on their different distribution in the core or shell regions of the composite nanofibers. ${ }^{8}$ It is known that PCL has a slow degradation rate in bulk objects as well as in nanofibers with nanopores on their surfaces. The release mechanism was governed by desorption or dissolution rather than a solid-state diffusion or degradation mechanism. ${ }^{7,19}$ Naproxen encapsulated directly by nanofibers could be released preferentially in the release media through desorption and diffusion from the nanofibers. However, rhodamine B embedded in nanoparticles suffered from two barriers, the nanoparticle and nanofiber, thus reducing the
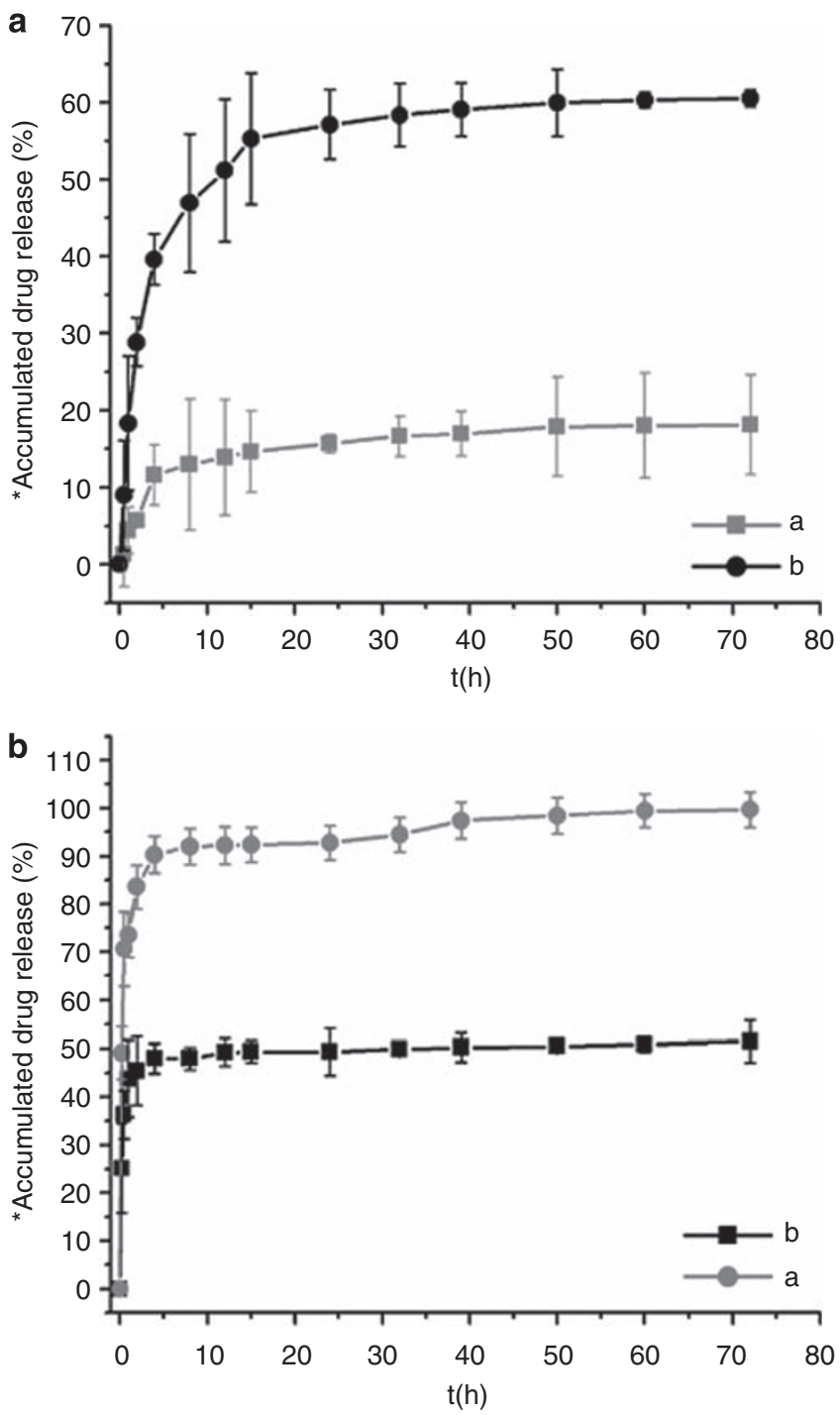

Figure 5 Release behaviors of rhodamine $B$ and naproxen from composite nanofibers NF-C (a) and NF-D (b). A, Rhodamine B; B, naproxen. ${ }^{*}$ Accumulated drug-release rate (\%) represents the ratio of the drug amount released from a formulation in a certain period of time to the total drug content in the formulation.

release rate. The release rate for the opposite loading case (that is, naproxen in CS nanoparticles and rhodamine B in PCL matrix) was shown in Figure 5b. There was obviously a burst release of naproxen at 
the initial stage, which might be attributed to the way the drug was encapsulated. Naproxen is highly insoluble in neutral and acidic solutions but is well dispersed in PBS and alkaline solution. The encapsulation efficiency of naproxen in CS CS was small, and naproxen may be encapsulated merely through adsorption or very weak interaction forces. Rhodamine B embedded in the PCL matrix also showed a fast release profile. Moreover, Srikar ${ }^{7}$ analyzed the factors influencing the release mechanism using desorption-limited theory and concluded that the chemical nature of the polymer and drug interaction were responsible for sorption-desorption processes in the presence of release media. Therefore, the different release profiles of naproxen from composite nanofibers NF-C and rhodamine B from NF-D may originate from the distinct physical and chemical properties of both drugs. ${ }^{8}$

It is important to note that the release behaviors of the drugs from composite nanofibers NF-C and NF-D, both of which can achieve a programmed release of dual drugs, were an indication of the varying properties of the systems. ${ }^{1,8}$ Composite nanofibers NF-C were designed to release naproxen at a rapid rate and rhodamine $\mathrm{B}$ at a slow rate. In nanofibers NF-D, we observed a faster release rate for rhodamine $\mathrm{B}$ than for naproxen; both had an obvious burst release, which could be ascribed to the compatibility between the drugs and carriers. There was no compatibility between rhodamine B and PCL because of their hydrophilic and hydrophobic properties, respectively, which caused the fast release of rhodamine B. Similarly, the incompatibility between CS and naproxen led to low encapsulation efficiency; moreover, naproxen leaked out from nanoparticles during stirring, causing quick release. In contrast, the good compatibility between naproxen and PCL resulted in a slower release rate than that of rhodamine B. It is known that drug release from nanofibers can be further controlled by adjusting the properties of the drug, the concentration or molecular weight of the polymer and the procedure used to prepare the electrospinning solution. ${ }^{8,27-30}$ As a result, more precise programmed drug delivery can be achieved through proper choices of polymer matrix or drugs to entrap within the core or shell region of nanofibers.

Figure 6 showed the cumulative release profiles of rhodamine $B$ from naked nanoparticles, electrospun nanofibers NF-B and composite nanofibers NF-C, The release rate of rhodamine B from bare nanoparticles was faster than that from the composite nanofibers NF-C, which agreed with the result reported by Qi et al. ${ }^{20}$ The barrier presented by the nanofiber and the prolonged release mechanism led to a slower drug-release rate from composite nanofibers NF-C. The release rate of rhodamine $B$ from nanofibers NF-B, in which rhodamine $B$ was embedded in PCL, was the fastest of all; the incompatibility between them may be the reason for the fast release rate. However, rhodamine B encapsulated by CS nanoparticles by means of electrostatic interaction exhibited a slow drug-release rate. In this case, it is believed that the release rate depended on the polymer-drug intermolecular interaction in the presence of release medium.

Figure 7 showed the release profiles of naproxen from electrospun nanofibers NF-A and composite nanofibers NF-C. The release of $39.60 \%$ naproxen from composite nanofibers NF-C was rapidly reached within $4 \mathrm{~h}$, as compared with $24.77 \%$ from nanofibers NF-A. This may have been caused by the different localization of naproxen in each of the nanofibers. Owing to the compatibility between the drug and polymer matrix, naproxen may have distributed uniformly by phase separation within nanofibers NF-A, hence, exhibiting a steady and constant slow release rate. ${ }^{31}$ However, when mixed with CS nanoparticles, the location of naproxen would be affected by the presence of the nanoparticles during electrospinning, possibly

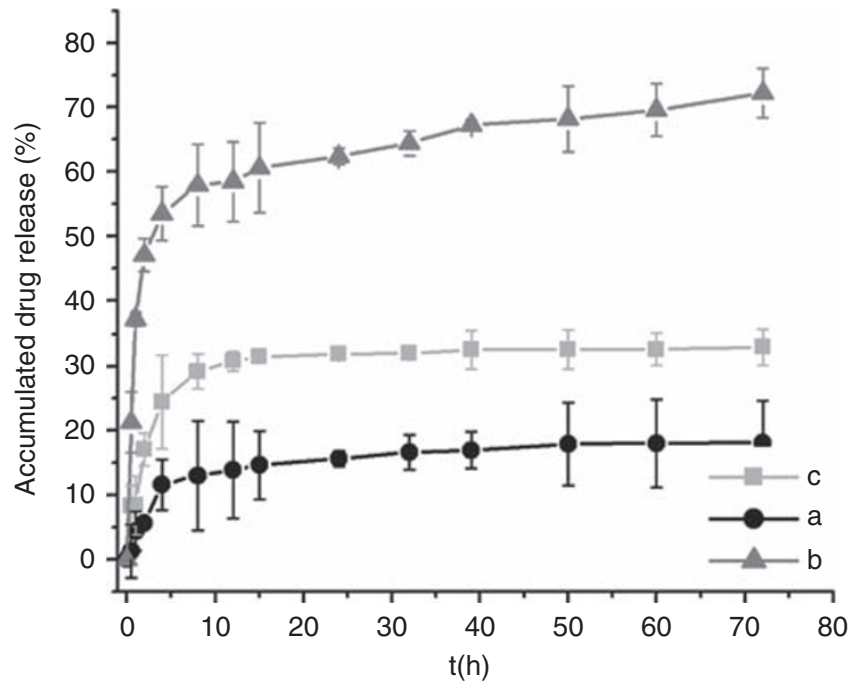

Figure 6 Release behaviors of rhodamine B from (a) composite nanofibers NF-C; (b) electrospun nanofibers NF-B; (c) bare nanoparticles.

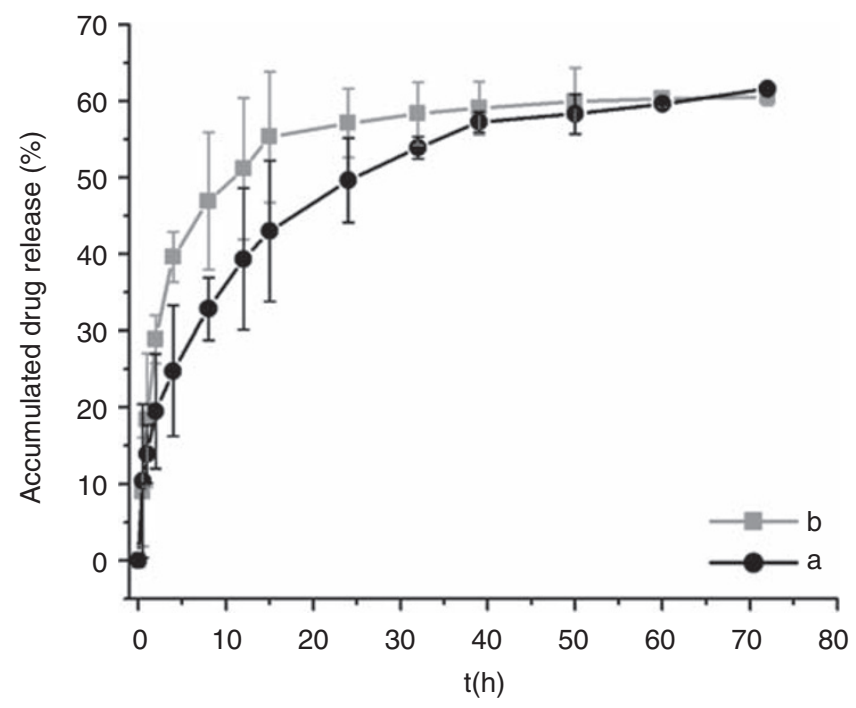

Figure 7 Release behaviors of naproxen from (a) composite nanofibers NF-C; (b) electrospun nanofibers NF-A.

because the nanoparticles could occupy the shell of the nanofibers, and the naproxen could situate itself near the surface of the composite nanofibers. Thus, naproxen could be released rapidly by overcoming the short diffusion pathway. ${ }^{9}$

\section{CONCLUSIONS}

In this study, we prepared core-shell structure composite nanofibers to encapsulate two distinct drugs by a single-nozzle step. First, singledrug-loaded nanoparticles were prepared with an average diameter of $380-500 \mathrm{~nm}$, and they were mixed with another single-drug-loaded polymer solution; the mixture was then electrospun. scanning electron microscope and LSCM images demonstrated that nanoparticles could be localized in the core region of electrospun nanofibers, forming a core-shell structure. Loading different drugs in the core and shell produced distinct release behaviors. A controlled release pattern for 
dual drugs was also achieved by adjusting the process used to prepare the electrospinning solution. Therefore, this method could provide a platform for dual-drug loading and programming the release of each drug, which is required in drug delivery systems and tissue engineering. As novel drug delivery systems, however, these core-shell structures present some problems that have not yet been resolved. Ideally, the nanoparticles should be well re-dispersed and distributed uniformly within the composite nanofibers. However, some particles cluster into agglomerates of different sizes, which may be overcome through further research. In addition, efficient drug loading is necessary, and this method must be improved. Furthermore, chemical drugs and biological macromolecules such as proteins and nucleic acids should also be investigated for encapsulation by composite nanofibers to extend their application in biomedical fields.

\section{ACKNOWLEDGEMENTS}

This study was supported by the Fundamental Research Funds for the Central Universities (Project No. CDJRC10230003) and National Natural Science Foundation of China (Project No. 30901977). The authors are grateful to Professor Li Yang and doctoral student Linhao Li for their experimental equipment and useful suggestions. The authors are also very thankful to Dr Gurinder K Singh for critically evaluating the manuscript.

1 Wei, M., Kang, B., Sung, C. M. \& Mead, J. Core-sheath structure in electrospun nanofibers from polymer blends. Macromol. Mater. Eng. 291, 1307-1314 (2006).

2 Taepaiboon, P., Rungsardthong, U. \& Supaphol, P. Vitamin-loaded electrospun cellulose acetate nanofiber mats as transdermal and dermal therapeutic agents of vitamin A acid and vitamin E. Eur. J. Pharm. Biopharm. 67, 387-397 (2007).

3 Angeles, M., Cheng, H. S. \& Velankar, S. S. Emusion electrospinning: composite fibers from drop breakup during electrospinning. Polym. Adv. Technol. 9, 728-733 (2008).

4 Yoo, H. S., Kim, T. G. \& Park, T. G. Surface-functionalized electrospun nanofibers for tissue engineering and drug delivery. Adv. Drug Deliv. Rev. 61, 1033-1042 (2009).

5 Chew, S. Y., Wen, Y., Dzenis, Y. \& Leong, K. W. The role of electrospinning in the emerging field of nanomedicine. Curr. Pharm. Des. 12, 4751-4770 (2006).

6 Sill, T. J. \& von Recum, H. A. Electrospinning: applications in drug delivery and tissue engineering. Biomaterials 29, 1989-2006 (2008).

7 Srikar, R., Yarin, A. L., Megaridis, C. M., Bazilevsky, A. V. \& Kelley, E. Desorptionlimited mechanism of release from polymer nanofibers. Langmuir 24, 965-974 (2008).

8 Jo, E., Lee, S., Kim, K. T., Won, Y. S., Kim, H. S., Cho, E. C. \& Jeong, U. Core-sheath nanofibers containing colloidal arrays in the core for programmable multi-agent delivery. Adv. Mater. 21, 968-972 (2009).

9 Jaklenec, A., Hinckfuss, A., Bilgen, B., Ciombor, D. M., Aaron, R. \& Mathiowitz, E. Sequential release of bioactive IGF-I and TGF-b1 from PLGA microsphere-based scaffolds. Biomaterials 29, 1518-1525 (2008)

10 Ahmed, F., Pakunlu, R. I., Brannan, A., Bates, F., Minko, T. \& Discher, D. E. Biodegradable polymersomes loaded with both paclitaxel and doxorubicin permeate and shrink tumors, inducing apoptosis in proportion to accumulated drug. J. Control. Release 116, 150-158 (2006).

11 Thakura, R. A., Florekb, C. A., Kohnb, J. \& Michniak, B. B. Electrospun nanofibrous polymeric scaffold with targeted drug release profiles for potential application as wound dressing. Int. J. Pharm. 364, 87-93 (2008).

12 Su, Y., Li, Q., Liu, S. P., Mo, X. \& Seeram, R. Controlled release of dual drugs from emulsion electrospun nanofibrous mats. Colloid Surf. B 73, 376-381 (2009).

13 Dong, B., Smith, M. E. \& Wnek, G. E. Encapsulation of multiple biological compounds within a single electrospun fiber. Small 5, 1508-1512 (2009).

14 Qi, L. F., Xu, Z. R., Jiang, X., Hu, C. H. \& Zou, X. F. Preparation and antibacterial activity of chitosan nanoparticles. Carbohyd. Res. 339, 2693-2700 (2004).

15 Papadimitriou, S., Bikiaris, D., Avgoustakis, K., Karavas, E. \& Georgarakis, M. Chitosan nanoparticles loaded with dorzolamide and pramipexole. Carbohyd. Polym. 73, 44-54 (2008).

16 Zhao, J. Y. \& Wu, J. M. Preparation and characterization of the fluorescent chitosan nanoparticle probe. Chin. J. Anal. Chem. 34, 1555-1559 (2006).

17 Zhang, Q. H., Zhen, J., Chang, Z. J., Zhu, M. F., Mo, X. M. \& Chen, D. J. Electrospun carbon nanotube composite nanofibres with uniaxially aligned arrays. Nanotechnology 18, 115611 (2007).

18 Shah, S., Pal, A., Kaushik, V. K. \& Devi, S. Preparation and characterization of venlafaxine hydrochloride-loaded chitosan nanoparticles and in vitro release of drug. J. Appl. Polym. Sci. 112, 2876-2887 (2009).

$19 \mathrm{Li}, \mathrm{H}$. \& Gao, C. Y. Preparation and properties of ionically cross-linked chitosan nanoparticles. Polym. Adv. Technol. 20, 613-619 (2009).

20 Qi, H. X., Hu, P., Xu, J. \& Wang, A. J. Encapsulation of drug reservoirs in fibers by emulsion electrospinning: morphology characterization and preliminary release assessment. Biomacromolecules 7, 2327-2330 (2006).

21 Saeed, K., Park, S. Y., Lee, H. J., Baek, J. B. \& Huh, W. S. Preparation of electrospun nanofibers of carbon nanotube/polycaprolactone nanocomposite. Polymer 47, 80198025 (2006).

22 Shao, C. L., Kim, H. Y., Gong, J., Ding, B., Lee, D. R. \& Park, S. J. Fiber mats of poly(vinyl alcohol)/silica composite via electrospinning. Mater. Lett. 57, 1579-1584 (2003).

23 Teo, W. E. \& Ramakrishna, S. Electrospun nanofibers as a platform for multifunctional, hierarchically organized nanocomposite. Comp. Sci. Technol. 69, 1804-1817 (2009).

24 Xu, X. L., Chen, X. S., Liu, A. X., Hong, Z. K. \& Jing, X. B. Electrospun poly(L-lactide)grafted hydroxyapatite/poly(L-lactide) nanocomposite fibers. Eur. Polym. J. 43, 3187-3196 (2007)

25 Xu, X. L., Chen, X. S., Ma, P. A., Wang, X. R. \& Jing, X. B. The release behavior of doxorubicin hydrochloride from medicated fibers prepared by emulsion-electrospinning. Eur. J. Pharm. Biopharm. 70, 165-170 (2008).

26 Lim, J. M., Moon, J. H., Yi, G. R., Heo, C. J. \& Yang, S. M. Fabrication of one-dimensional colloidal assemblies from electrospun nanofibers. Langmuir 22 3345-3349 (2006).

27 Tungprapa, S., Jangchud, I. \& Supaphol, P. Release characteristics of four model drugs from drug-loaded electrospun cellulose acetate fiber mats. Polymer 48, 5030-5041 (2007).

28 Gandhi, M., Srikar, R., Yarin, A. L., Megaridis, C. M. \& Gemeinhart, R. A. Mechanistic examination of protein release from polymer nanofibers. Mol. Pharm. 6, 641-647 (2009).

29 Hughes, G. A. Nanostructure-mediated drug delivery. Nanomedicine 1, 22-30 (2005).

30 Taepaiboon, P., Rungsardthong, U. \& Supaphol, P. Drug-loaded electrospun mats of poly(vinyl alcohol) fibers and their release characteristics of four model drugs. Nanotechnology 17, 2317-2329 (2006).

31 Zeng, J., Yang, L. X., Liang, Q. Z., Zhang, X. F., Guan, H. L., Xu, X. L., Chen, X. S. \& Jing, X. B. Influence of the drug compatibility with polymer solution on the release kinetics of electrospun fiber formulation. J. Control Release 105, 43-51 (2005).

\section{APPENDIX}

Ultraviolet calibration curves of rhodamine B and naproxen in phosphate buffer saline $(\mathrm{pH}=7.4)$.

Supplementary Information accompanies the paper on Polymer Journal website (http://www.nature.com/pj) 